\title{
Flexible Tactile Sensing Arrays for Robotics: Architectural Robustness and Yield Considerations
}

\author{
Phillip W. Barth ${ }^{*}$, Mark J. Zdeblick, Zenon Kuc, and Patricia A. Beck \\ Center for Integrated Systems \\ Stanford University \\ Stanford, California 94305 \\ ${ }^{*}$ Present address: \\ NovaSensor \\ 2975 Bowers Avenue \\ Santa Clara, CA 95050
}

The Robot Skin project at Stanford is an attempt to create new tactile sensing technology for general-purpose robot manipulators. Such manipulators, designed to be functionally equivalent (at least) to the human hand, will require sensor arrays that fulfill several requirements: (1) many sensor elements (on the order of 64-256) to minimize tactile imaging time, (2) close spacing of small sensing elements for fine spatial discrimination, (3) ability to be molded to a convex fingertip surface for taction on concave surfaces, (4) ability to sense both force and temperature in order to fully mimic biological touch,(5) tolerance to over-scale forces without damage, and (6) "graceful" failure in use, i.e., functional survival of most of the array when part of it is damaged. In addition to the above, which are considered requirements, several other characteristics are desirable. Foremost is the ability to sense shear forces, which provide information on actual or incipient slip of a grasped object. It also appears desirable for tactile sensor arrays to possess some local pattern-processing capability which can lower the transmission bandwidth required to the robot's central information processor. In order for such sensor arrays to be useful in practical systems, cost constraints must also be considered; such constraints argue for automated and/or batch fabrication of the arrays. Finally, given the differences between biology and various non-biological technologies, it may be possible to achieve functionality not available to biological touch, e.g., by including capacitive proximity sensing capability in such arrays.

No tactile sensor array developed to date fills all, nor even most, of these requirements and desires well. Several investigators have recently developed tactile sensing arrays suitable for imaging shapes on hard, flat surfaces (e.g., [1-3]), but not well suited for taction on curved surfaces. Others have developed single tactile sensors suitable for incorporation into arrays (e.g., [4]), which will probably prove uneconomical when large numbers of sensors are needed. Still others have developed flexible capacitive arrays [5-6] suitable for use on curved surfaces but incapable of obtaining temperature or proximity data. The purpose of the present effort is to develop a technology base suitable to the achievement of all of the above goals, and to incrementally demonstrate useful steps toward those goals.

The proposed physical structure of such arrays, based on previously developed technology for linear thermometer arrays [7], places silicon islands in a flexible polyimide substrate (Figure 1). The islands are interconnected by leads embedded in the substrate; each island will contain one or more force sensors, and may also contain temperature and proximity sensors. To date the island shapes have been successfully fabricated in polyimide substrates for one-sensor islands (suitable for normal force sensing) and for four-sensor islands (suitable for sensing both shear and normal forces), both of these in $5 \times 5$ arrays. Complete process integration in a purely passive force sensor array is under way for these designs. Eventually $16 \times 16$ arrays with active signal processing are contemplated.

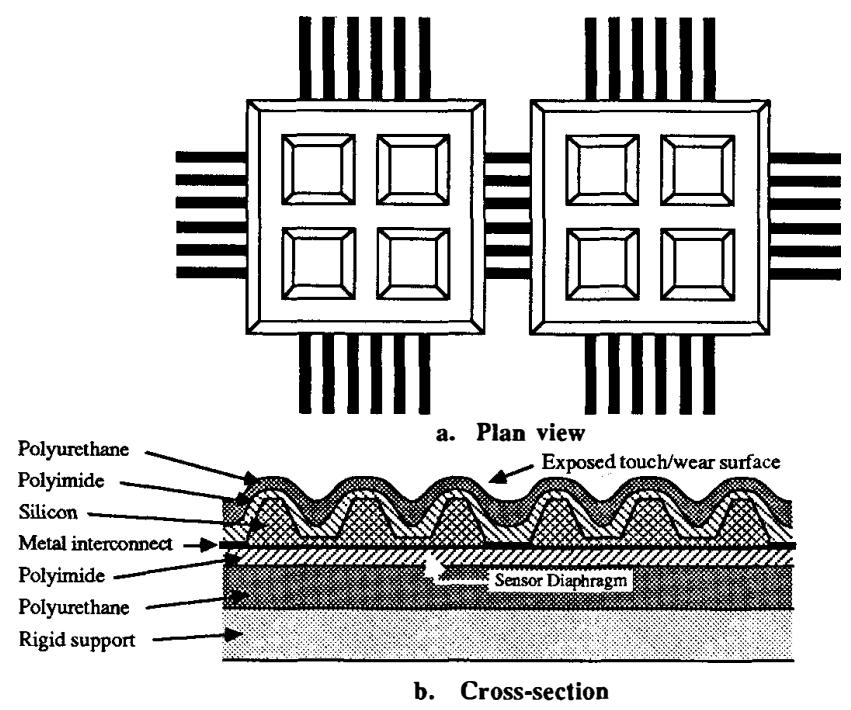

Figure 1. Adjacent sensor islands with interconnections

In service these arrays will be subject to wear and tear in the same way that biological skin is, but without the self-repair capability of living tissue. Leads will break between islands, islands will be crushed and broken, and eventually the array must be replaced. To delay replacement as long as possible, robust physical and electronic architectures are needed.

Sensor placement on each island can be either near the perimeter of the island or internal to the island, and has a strong effect on physical robustness. Initial designs placed sensors on flaps near the island edges. This arrangement leads to high sensitivity because loading forces are largest at island edges. However, computer modeling predicts a non-linear voltage-versus-force response because of compression effects in the underlying support layer, making it difficult to detect normal and shear forces separately. In addition, the flap arrangement is expected to result in fragility because wires embedded in the polyimide will have little strain relief at the island edges, and because the flaps will be subject to the maximum stress occurring anywhere on the island. Internal placement of the sensors results in a linear response, maximum polyimide thickness at island edges for good strain relief of the leads, and uniform loading over most of the island.

Electronic robustness is ensured by choosing the most fault-tolerant addressing scheme consistent with addressing time and silicon area availability. Possible addressing schemes including the following: one hard-wire connection to each sensor, one wire to all sensors with sequential or serial-address readout, row-column addressing with and without redundant wires, parallel-to-serial shift register readout, and a "global bus" mesh with serial or parallel addressing. 
The most promising architectures appear to be row-column address schemes with redundancy; such schemes provide near-optimum robustness with minimal circuit area and fast address times. A computer simulation of wear, in the form of random cuts occurring over time in the interconnections, has been developed and applied to row-column address schemes of several types, and shows that closely-spaced redundant wires aid in achieving robustness (Figure 2).

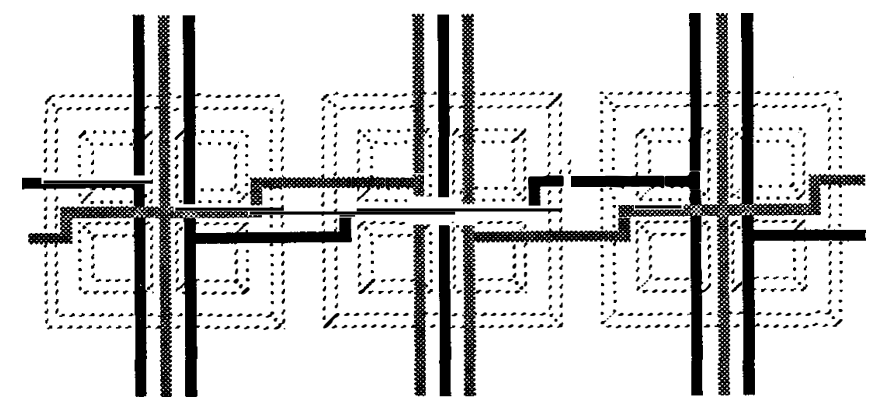

Figure 2. Triply-redundant column addressing with single-pixel separation of redundant lines

The designs presently being constructed use conventional diaphragm fabrication by etching from the wafer backside. The yield-reducing problems with this type of technology are well known. In addition, for the present application where each island is effectively a force sensor rather than a pressure sensor, the diaphragms fabricated by backside etching may be subject to damage from pointed objects. Purely front-sided sensor fabrication, like that advocated by Guckel (e.g.,[8]), offers the possibility of higher yield and greater robustness. We are pursuing a modification of previous techniques for fabricating sensor diaphragms out of polycrystalline silicon, with the goal of creating capacitive force sensors (Figure 3 ) for the next generation of tactile sensing arrays. The modified fabrication techniques allow etching of a sacrificial spacer layer beneath the diaphragm through an array of holes which covers the entire diaphragm area, as opposed to etching from only the edges of the diaphragm. The technique should greatly shorten the etch times required, and so permit insulating standoffs necessary for the capacitor structure to remain in place.

Any flexible array containing closely-spaced rigid islands in a rectilinear arrangement is flexible only along the rows and columns between islands. When such arrays are placed on robot fingertips this limits the fingertip shapes to variations on the theme shown in Figure 4, where coverage of most of the fingertip is achieved by bending the array along two axes. This arrangement leaves a "blind spot" which will probably be acceptable in most applications. The blind spot can be eliminated by extra care in layout of the array and shaping of the fingertip, but such steps will add extra cost and will probably not be justified in most cases.

\section{Acknowledgement}

This work has been sponsored by the NASA Ames Research Center and by the NASA-funded Center for Aeronautics and Space Information Sciences at Stanford.

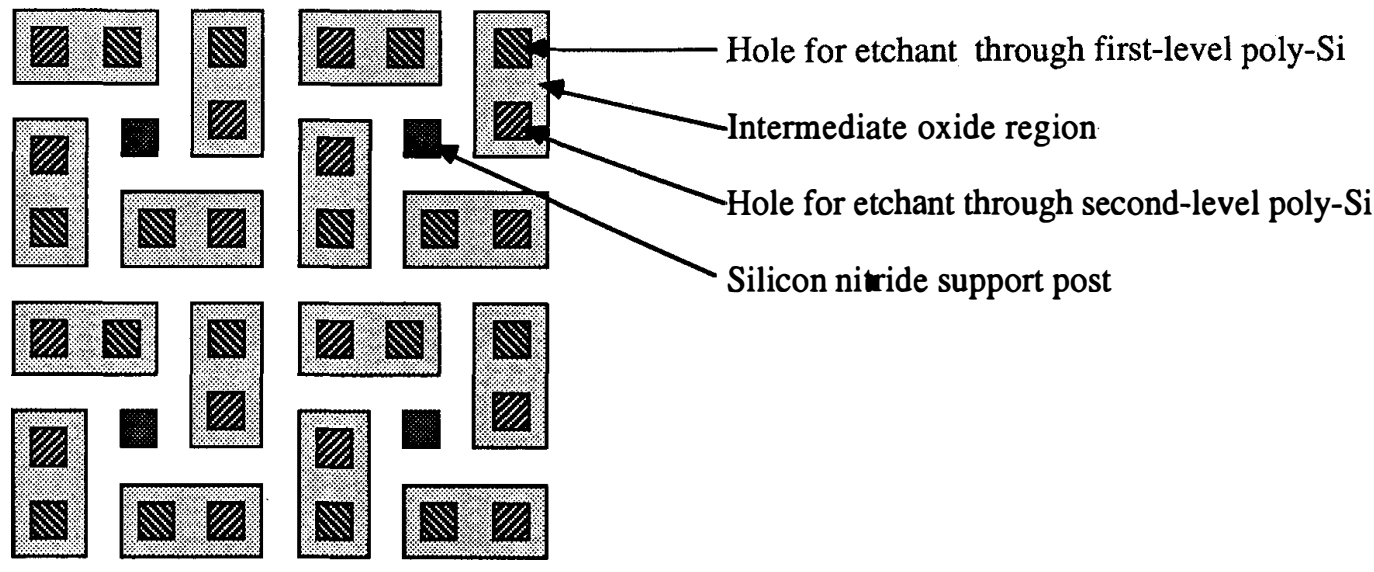

a. Plan view

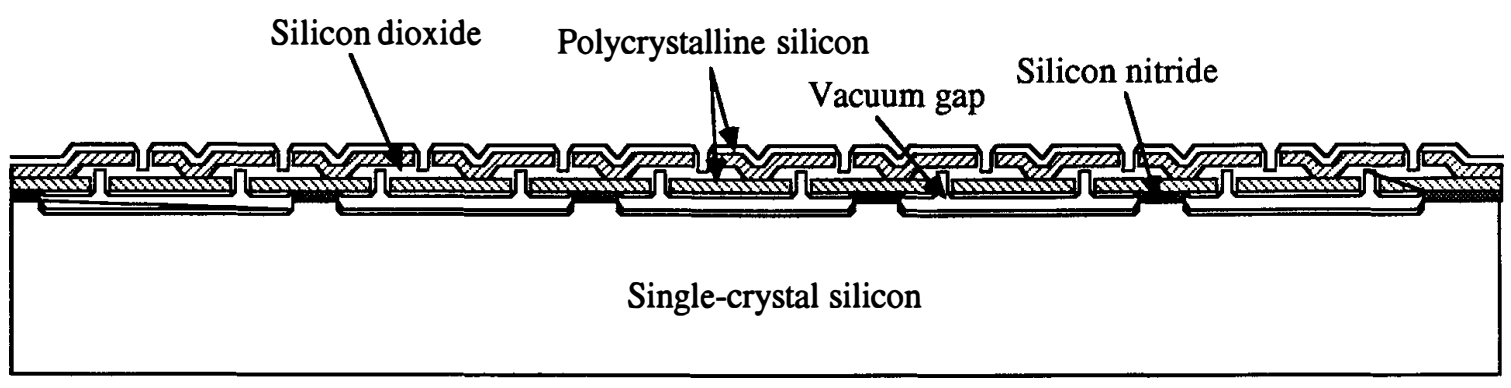

b. Cross-section

Figure 3. Two-level Polysilicon Diaphragm Sructure 


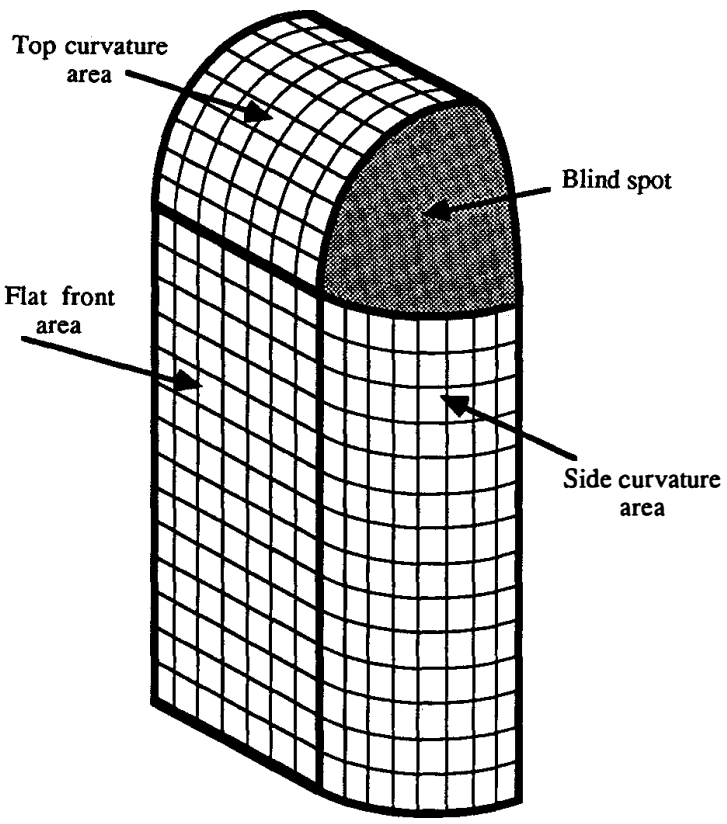

Figure 4. Tactile array attachment to a fingertip (two axes of bend available)

\section{References}

[1] R.M. White and A. King, "Tactile Array for Robotics Employing a Rubbery Skin and a Solid-State Optical Sensor," paper 2.1, Transducers '85, Digest of Technical Papers, IEEE Catalog No. 85CH2127-9, pp 18-21, June 1985.

[2] K.J. Chun and K.D. Wise, "A Capacitive Silicon Tactile Imaging Array," paper 2.2, Transducers '85, Digest of Technical Papers, IEEE Catalog No. 85CH2127-9, pp 22-25, June 1985.

[3] K. Wong and J. Van der Spiegel, "A Shielded Piezoresistive Tactile Sensor Array," paper 2.3, Transducers '85, Digest of Technical Papers, IEEE Catalog No. 85CH2127-9, pp 26-28, June 1985.

[4] K. Petersen, C. Kowalski, J. Brown, H. Allen, and J. Knutti, "A Force-Sensing Chip Designed for Robotic and Manufacturing Automation Applications," paper 2.4, Transducers '85, Digest of Technical Papers, IEEE Catalog No. 85CH2127-9, pp 30-32, June 1985.

[5] Ron Fearing, Artificial Intelligence Laboratory, Stanford University Computer Science Department. (Unpublished)

[6] Michael R. Neuman and Chung-Chiun Liu, "Fabrication of Biomedical Sensors Using Thin and Thick Film Microelectronic Technology," Micromachining and Micropackaging of Transducers, Clifford Fung, et al, editors, Elsevier Science Publishing Co. Inc., New York, 1985. ISBN 0-444-42560-8. pp 225-238.

[7] Phillip W. Barth, Sharon L. Bernard, and James B. Angell "Flexible Circuit and Sensor Arrays Fabricated by Monolithic Silicon Technology," IEEE Transactions on Electron Devices, Vol. Ed-32, No. 7, pp 1202-1205, July 1985.

[8] H. Guckel, D.W. Burns, H.H. Busta, and J.F. Detry, "LaserRecrystallized Piezoresistive Micro-Diaphragm Sensors," 1985 Conference on Solid-State Sensors and Actuators, Digest of Technical Papers, IEEE Catalog No. 84-62799, pp 182-185. 